\title{
EL CÁNCER DE PRÓSTATA EN LA COMUNIDAD DE MADRID EN EL AÑO 2000. I - INCIDENCIA(*)
}

\author{
F. HERRANZ AMO ${ }^{1}$, F. ARIAS FUNEZ ${ }^{2}$, M. ARRIZABALAGA MORENO ${ }^{3}$, \\ F.J. CALAHORRA FERNÁNDEZ ${ }^{4}$, J. CARBALLIDO RODRIGGUEZ ${ }^{5}$, R. DIZ RODRÍGUEZ ${ }^{6}$, \\ J.A. HERRERO PAYO ${ }^{7}$, C. LLORENTE ABARCA ${ }^{8}$, J.C. MARTÍN MARTÍNEZ ${ }^{9}$, \\ L. MARTÍNEZ-PIÑEIRO LORENZO ${ }^{10}$, R. MÍNGUEZ MARTÍNEZ ${ }^{11}, J^{\prime}$. MORENO \\ SIERRA $^{12}$, A. RODRÍGUEZ ANTOLÍN ${ }^{13}$, J.C. TAMAYO RUIZ ${ }^{14}$, J. TURO ANTONA ${ }^{15}$
}

${ }^{1}$ H.G.U. Gregorio Marañón. ${ }^{2} \mathrm{H}$. Ramón y Cajal. ${ }^{3} \mathrm{H}$. de Móstoles. ${ }^{4}$ Fundación Jiménez Díaz. ${ }^{5} \mathrm{H}$. Puerta de Hierro. ${ }^{6} \mathrm{H}$. del Aire. ${ }^{7} \mathrm{H}$. Severo Ochoa. ${ }^{8} \mathrm{H}$. Fundación de Alcorcón. ${ }^{9} \mathrm{H}$. El Escorial. ${ }^{10} \mathrm{H}$. de La Paz. ${ }^{11} \mathrm{H}$. de La Princesa. ${ }^{12} \mathrm{H}$. Clínico. ${ }^{13} \mathrm{H}$. 12 de Octubre. ${ }^{14} \mathrm{H}$. Príncipe de Asturias $y{ }^{15} \mathrm{H}$. Militar Gómez Ulla. Madrid.

Actas Urol Esp. 27 (5): 323-334, 2003

\section{RESUMEN}

"EL CÁNCER DE PRÓSTATA EN LA COMUNIDAD DE MADRID EN EL AÑO 2000. I - INCIDENCIA(*) OBJETIVO: Conocer la incidencia del cáncer de próstata en la Comunidad de Madrid y en sus distintas Áreas Sanitarias en el año 2000.

MATERIAL Y MÉTODO: Estudio de casos incidentes con confirmación histológica de cáncer de próstata y con recogida de datos retrospectiva en la Comunidad de Madrid durante el año 2000, tanto en la Sanidad Pública como en la Privada.

RESULTADOS: La incidencia bruta del cáncer de próstata en la Comunidad de Madrid fue de 100,4 casos por 100.000 hombres. La incidencia ajustada a la población española, europea y mundial fue de $120,1,103,5$ y 68,6 casos por 100.000 hombres respectivamente. La edad media al diagnóstico fue de $70 \pm 7,8$ (40-94) años con una mediana de 70 años. El tramo etario con una incidencia más elevada se situó entre los 70 y los 79 años.

CONCLUSIONES: La incidencia del cáncer de próstata en la Comunidad de Madrid es más baja que en USA, pero más elevada que en la inmensa mayoría de los países o regiones de Europa. El diferente uso del PSA en las distintas Áreas Sanitarias de la Comunidad podría justificar las diferencias observadas en la incidencia por Área.

PALABRAS CLAVES: Cáncer de próstata. Incidencia. Comunidad de Madrid.

\section{ABSTRACT}

"THE PROSTATE CANCER IN THE COMMUNITY OF MADRID IN 2000. I.- INCIDENCE"

OBJECTIVE: To know the incidence in the year 2000 of prostate cancer in the Autonomous Community of Madrid and its breakdown by Health Areas.

MATERIAL AND METHOD: Study of histologically confirmed prostate cancer case reports and retrospective data acquisition for 2000 in the Autonomous Community of Madrid, both from Public and Private Health Care hospitals.

RESULTS: Gross incidence of prostate cancer in the Autonomous Community of Madrid was 100.4 cases per 100,000 males. The incidence adjusted for the Spanish, European and Worldwide population was 120.1, 103.5 and 68.6 cases per 100,000 males, respectively. Mean age at diagnosis was $70 \pm 7.8(40-94)$ years, median of 70 years. The age group with higher incidence was 70 to 79 years.

CONCLUSIONS: The incidence of prostate cancer in the Autonomous Community of Madrid is lower than that in the US but higher than in most countries or regions in the EU. The different way of using PSA testing in the Health Areas of the Autonomous Community may explain the differences seen in terms of incidence by Area.

KEY WORDS: Prostate cancer. Incidence. Community of Madrid.

$\left({ }^{*}\right)$ Este artículo forma parte del estudio "El cáncer de próstata en la Comunidad de Madrid en el año 2000", auspiciado por la Sociedad Urológica Madrileña y financiado por AstraZéneca. 
$\mathrm{E}^{1}$ cáncer de próstata (CP) es la neoplasia no cutánea más frecuentemente diagnosticada en USA y la segunda causa de muerte por enfermedad oncológica después del cáncer de pulmón. Se estima que en el año 2001 se diagnosticaron en USA 198.100 nuevos casos y murieron por esta enfermedad 31.500 varones $^{1}$. La probabilidad de desarrollar un CP aumenta con la edad, estimándose en un 17\% ( 1 de cada 6 hombres) la posibilidad de desarrollarlo a lo largo de la vida.

En un estudio recientemente publicado sobre la estimación de la incidencia del cáncer en Europa en $1995^{2}$, el CP en el varón se sitúa en el 3o lugar en frecuencia (11\%) después de las neoplasias de pulmón (22\%) y colorectal (12\%). En los países de la Unión Europea el CP ocupa el segundo puesto en incidencia después del cáncer de pulmón y por delante del colorectal. La estimación de la incidencia del CP en Europa fue de 47,4 casos y de 55,5 casos por 100.000 hombres en la Unión Europea. Existe una gran variabilidad entre los distintos países, así Islandia tiene una incidencia de 124,5 frente a 16,7 casos por 100.000 hombres en la República de Moldavia. Para España la incidencia estimada fue de 33,9 casos por 100.000 hombres, basándose en los registros de tumores de: "Albacete, Gerona, Granada, La Rioja, Murcia, Asturias, Mallorca, Navarra, Guipúzcoa, Canarias, Tarragona, Euskadi y Zaragoza".

En España la incidencia del CP es desconocida. Los únicos datos disponibles se basan en registros de tumores locales cuyos datos publicados tienen un desfase de más de una década ${ }^{3,4}$. Estos datos corresponden a la era pre-PSA y por lo tanto no pueden ser representativos de la incidencia actual del CP.

En la Comunidad de Madrid existe un registro de tumores dependiente de la Consejería de Sanidad, pero hasta la fecha no ha comunicado datos sobre la incidencia del CP. En 1997 se publicó un estudio donde se detallaba la evolución de la incidencia el $\mathrm{CP}$ en los municipios madrileños de Móstoles y Alcorcón en el periodo 1983-19965 , en este estudio se observó un incremento de la incidencia siendo en 1996 de 36,5 casos por 100.000 hombres.

El cáncer de próstata debido a su elevada incidencia y prevalencia, a la morbilidad que origina la enfermedad por sí mismo y a la derivada de su tratamiento y al elevado coste que supone su tratamiento, representa un importante problema de salud pública.

El objetivo principal de este estudio fue conocer la incidencia del CP en la Comunidad de Madrid.

\section{MATERIAL Y MÉTODO Ámbito del estudio ${ }^{6}$}

La Comunidad de Madrid (CM) ocupa una extensión de $8.029 \mathrm{~km}^{2}$, lo que representa el $1,6 \%$ de la superficie total de España. Según el padrón municipal de habitantes de 1998 (http://www.comadrid.es/iestadis/pc98.htm) la población de la $\mathrm{CM}$ ascendía a 5.091.336 habitantes, con una densidad de 634 habitantes por $\mathrm{km}^{2}$, de los cuales el $48 \%$ son hombres y el $52 \%$ mujeres. La CM es, administrativamente, una Comunidad Autónoma uniprovincial dividida en 179 municipios; de ellos, 27 forman la llamada corona metropolitana, que junto a la capital conforman el área metropolitana de Madrid, donde se concentra el $91 \%$ de la población en menos del $25 \%$ de la extensión de la región.

La evolución demográfica de la CM ha superado su fase más expansiva. En el último periodo intercensal, 1991-1996, sólo aumentó su población en un 1,5\%. Este crecimiento se distribuyó de forma muy irregular; el municipio de Madrid perdió el $4,77 \%$, la corona metropolitana aumentó en un $8,1 \%$ y los municipios no metropolitanos presentaron un crecimiento del 25,5\% de su población. Como el resto de España está experimentando un proceso de envejecimiento demográfico. En el último periodo intercensal el grupo de personas con 65 años o más aumentó en casi 100.000 personas, mientras que la población en su conjunto creció sólo en 74.000 habitantes.

La división territorial sanitaria vigente se reguló por el Decreto 187/98 de 5 de noviembre, que estableció 11 áreas sanitarias, 34 distritos sanitarios y 247 zonas básicas de salud. La configuración de las áreas sanitarias de la CM mantiene una estructura radial, en función de las principales instalaciones sanitarias de la región, localizadas en torno a la capital y a los núcleos con mayor número de habitantes. De este modo la mayoría de las áreas de salud engloban en su territorio zonas urbanas y rurales (Fig. 1). La dis- 


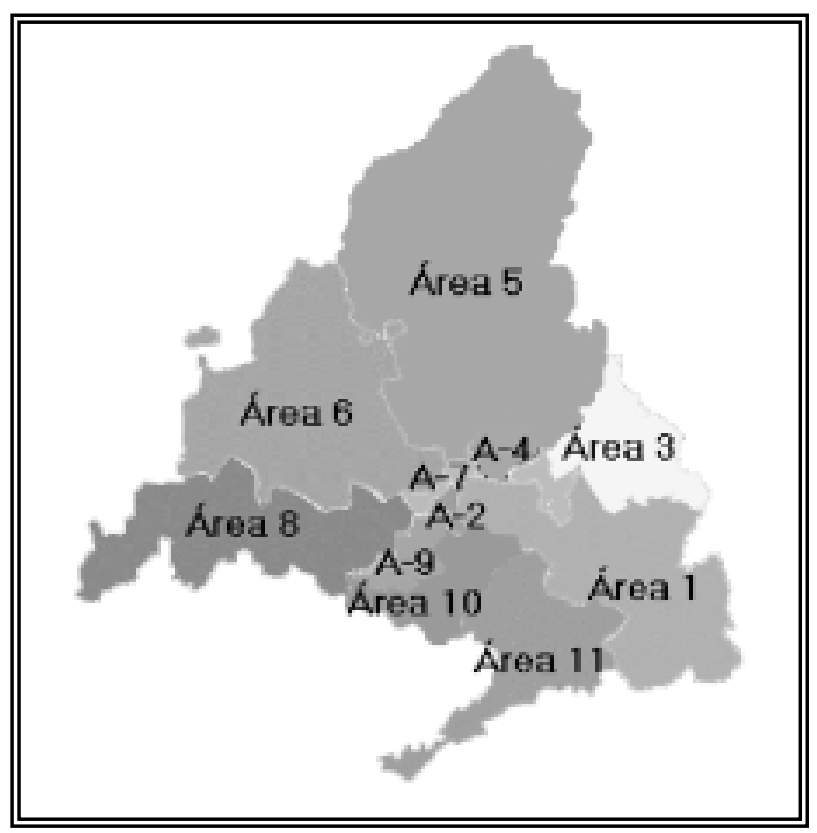

FIGURA 1. Mapa Sanitario de la Comunidad de Madrid.

tribución de la población según las distintas Áreas Sanitarias se detalla en la Tabla I. En la Tabla II se detallan los Hospitales (con Servicio de Urología de adultos) que atienden a la población de cada área Sanitaria. Los hospitales de la Sanidad Militar (Gómez Ulla y del Aire), la Fundación Jiménez Díaz y el Hospital de la Cruz Roja no tienen un Área Sanitaria asignada. Los hospitales de la Sanidad Militar atienden a la población militar de la CM. El Hospital de la Cruz Roja, en su labor urológica, atiende el exceso de lista de espera quirúrgica de otros Centros. La Fundación Jiménez Díaz tenía en el año 2000 un concierto para atender a los pacientes de los distritos Centro (Área 7) y Arganzuela (Área 11).

\section{Metodología}

\section{Objetivos}

El objetivo principal del estudio fue conocer la incidencia de cáncer de próstata con confirmación histológica en la CM durante el año 2000. Como objetivo secundario nos planteamos conocer la situación del CP desde el punto de vista de presentación, diagnóstico, etc. en nuestra Comunidad.

\section{Población a estudiar}

Los sujetos del estudio fueron la población masculina de la CM diagnosticadas de CP en el año 2000. No se realizó ningún muestreo y se intentaron recoger todos los casos. Como no fue posible, se estimó la incidencia de las Áreas que no participaron en base a las Áreas con una distribución similar de la población masculina.

Diseño del estudio y variables a estudiar

Es un estudio de casos incidentes con recogida de datos retrospectiva. Las variables de estudio fueron: edad, patología asociada, forma de

\section{TABLA I}

POBLACIÓN DE LA COMUNIDAD DE MADRID POR ÁREAS SANITARIAS, CON ESPECIAL ATENCIÓN A LOS HOMBRES DE MÁS DE 40 AÑOS. (FUENTE: PADRÓN A 1 DE ENERO DE 1998.

En: http:/www.comadrid.es/iestadis/pc98.htm)

\begin{tabular}{|c|c|c|c|}
\hline Área sanitaria & Población & Hombres (\%) & Hombres >40 años (\%) \\
\hline A1 & 647.154 & $310.199(47,9 \%)$ & $127.534(41,1 \%)$ \\
\hline A2 & 404.828 & $186.690(46,1 \%)$ & $79.606(42,6 \%)$ \\
\hline A3 & 273.351 & $134.297(49,1 \%)$ & $48.874(36,4 \%)$ \\
\hline A4 & 510.576 & $242.392(47,5 \%)$ & $104.672(43,2 \%)$ \\
\hline A5 & 620.405 & $298.843(48,2 \%)$ & $121.458(40,6 \%)$ \\
\hline A6 & 436.166 & $212.236(48,7 \%)$ & $80.815(38,1 \%)$ \\
\hline A7 & 521.471 & $238.640(45,8 \%)$ & $109.764(46,0 \%)$ \\
\hline A8 & 403.412 & $200.789(49,8 \%)$ & $77.833(38,8 \%)$ \\
\hline A9 & 349.904 & $175.548(50,2 \%)$ & $61.403(35,0 \%)$ \\
\hline A10 & 254.213 & $126.788(49,9 \%)$ & $47.804(37,7 \%)$ \\
\hline A11 & 669.856 & $317.845(47,4 \%)$ & $136.437(42,9 \%)$ \\
\hline Total & $\mathbf{5 . 0 9 1 . 3 3 6}$ & $\mathbf{2 . 4 4 4 . 2 6 7 ( 4 8 \% )}$ & $\mathbf{9 9 6 . 2 0 0 ~ ( 4 0 , 8 \% )}$ \\
\hline
\end{tabular}


TABLA II

RELACIÓN DE HOSPITALES DE LA RED ASISTENCIAL PÚBLICA SEGÚN LAS DISTINTAS ÁREAS SANITARIAS DE LA COMUNIDAD DE MADRID

\begin{tabular}{|c|c|c|}
\hline $\begin{array}{l}\text { Área } \\
\text { sanitaria }\end{array}$ & \begin{tabular}{|l|} 
Distritos \\
sanitarios
\end{tabular} & Hospitales públicos \\
\hline $\mathrm{Al}$ & $\begin{array}{l}\text { Arganda } \\
\text { Moratalaz } \\
\text { Retiro } \\
\text { Vallecas }\end{array}$ & H.G.U. Gregorio Marañón \\
\hline $\mathrm{A} 2$ & $\begin{array}{l}\text { Coslada } \\
\text { Salamanca } \\
\text { Chamartín }\end{array}$ & H.U. de La Princesa \\
\hline A3 & $\begin{array}{l}\text { Alcalá de Henares } \\
\text { Torrejón de Ardoz }\end{array}$ & H.U. Principe de Asturias \\
\hline A4 & $\begin{array}{l}\text { Ciudad Lineal } \\
\text { San Blas } \\
\text { Hortaleza }\end{array}$ & H. Ramón y Cajal \\
\hline A5 & $\begin{array}{l}\text { Alcobendas } \\
\text { Colmenar Viejo } \\
\text { Tetuán } \\
\text { Fuencarral }\end{array}$ & H. La Paz \\
\hline A6 & $\begin{array}{l}\text { Majadahonda } \\
\text { Collado Villalba } \\
\text { Moncloa }\end{array}$ & $\begin{array}{l}\text { Clínica Puerta de Hierro } \\
\text { H. de El Escorial }\end{array}$ \\
\hline A7 & $\begin{array}{l}\text { Centro } \\
\text { Chamberí } \\
\text { Latina }\end{array}$ & H. Clínico Univ. de San Carlos \\
\hline A8 & $\begin{array}{l}\text { Móstoles } \\
\text { Alcorcón } \\
\text { Navalcarnero }\end{array}$ & $\begin{array}{l}\text { Fundación Hospital Alcorcón } \\
\text { H. de Móstoles }\end{array}$ \\
\hline A9 & $\begin{array}{l}\text { Leganés } \\
\text { Fuenlabrada }\end{array}$ & H. Severo Ochoa \\
\hline $\mathrm{A} 10$ & $\begin{array}{l}\text { Parla } \\
\text { Getafe }\end{array}$ & H. Universitario de Getafe \\
\hline Al1 & $\begin{array}{l}\text { Aranjuez } \\
\text { Arganzuela } \\
\text { Villaverde } \\
\text { Carabanchel } \\
\text { Usera }\end{array}$ & H. 12 de Octubre \\
\hline
\end{tabular}

presentación, tacto rectal, PSA, método de diagnóstico, score de Gleason, estudio de extensión tumoral, estadio tumoral y tratamiento aplicado.

\section{Esquema de trabajo}

El Coordinador del Estudio contactó con los Servicios de Urología de todos los Hospitales Públicos de la CM. Los Servicios de Urología participantes nombraron un Investigador (Urólogo) cuya misión fue:
1. Identificación de los sujetos con CP diagnosticados en el año 2000 en la base de datos de los Servicios de Anatomía Patológica de cada Centro Hospitalario.

2. Revisión de las $\mathrm{H}^{\mathrm{a}}$. Clínicas y recogida de datos.

3. Envío de las hojas de recogida de datos debidamente cumplimentadas al Coordinador.

El Coordinador revisó y comprobó la calidad de los datos, recabando la información necesaria en aquellos casos en que los datos fueron incompletos o incongruentes. También construyó, introdujo y depuró la base de datos con todos los pacientes incluidos en el estudio.

En la Sanidad Privada, debido a las peculiaridades de la misma (gran dispersión de los pacientes, difícil acceso a las $\mathrm{H}^{\mathrm{a}}$.C., etc.) no fue posible seguir el mismo esquema de trabajo. Según los datos de un estudio piloto realizado con los sujetos diagnosticados de CP en el Área 1, el 96,5\% de los mismos fueron diagnosticados mediante biopsia transrectal. Por lo tanto, el Coordinador contactó con los Urólogos que realizan biopsia transrectal ecodirigida de la próstata en el desarrollo de su actividad profesional privada. En estos casos sólo se les solicitó la identificación de los pacientes con CP diagnosticados en el año 2000.

Una vez comprobado que no existía duplicidad de casos, se suprimió el campo de todos los registros que contenía la identificación personal, para cumplir con la Ley Orgánica 15/1999, de Protección de Datos de Carácter Personal (BOE, núm. 298, de 14 de diciembre de 1999).

\section{Análisis estadístico}

En las áreas participantes en el estudio se calcularon sus tasas brutas expresándolas como número de pacientes con cáncer de próstata por 100.000 varones y específicas por grupos de edad, expresándolas como número de pacientes con cáncer de próstata dentro de un grupo de edad determinado por 100.000 varones de ese mismo grupo etario. La incidencia de las áreas que no participaron se estimaron en función de las áreas participantes con una estructura etaria semejante. La tasa bruta de la CM se obtuvo sumando los casos de $\mathrm{CP}$ de las áreas participantes con los casos estimados de las áreas que 
no participaron y con los casos diagnosticados en la Sanidad Privada, dividiéndolo por la población en riesgo. Para comparar la incidencia en las distintas Áreas Sanitarias se calculó la incidencia ajustada según la edad para cada Área.

Para el cálculo de la incidencia se han utilizado los datos poblacionales de la revisión del padrón a 1 de enero de 1998 (http://www.comadrid.es/iestadis/pc98.htm). El cálculo de la incidencia ajustada por la edad se ha realizado según el método directo ${ }^{7}$. La incidencia bruta de la CM se ajustó a la población española de 1998 (Fuente: Instituto Nacional de Estadística. http://www. ine.es/ mbase/cgi/um. Revisión del Padrón Municipal de 1998) y a la población estandarizada europea y mundial (Fuente: http://www-dep.iarc.fr/eucan/ eucan.htm).

Antes de que los Investigadores pudieran comparar los datos de sus respectivas áreas con las demás, contestaron una encuesta relacionada con los criterios existentes en sus respectivos hospitales para el diagnóstico (uso libre o restringido según edad del PSA y biopsia prostática), estudio de extensión tumoral (pruebas realizadas en función de variables pronósticas de metástasis) y tratamiento del CP.

\section{RESULTADOS}

De los 17 Hospitales con Servicio de Urología existentes en la Comunidad de Madrid, 15 han participado en el estudio (Tabla III); obteniéndose información de 10 de las 11 Áreas Sanitarias. Los casos de CP del Área 10, que no participó, se estimaron utilizando para ello las Áreas 3, 6 y 8 que presentan una distribución similar de la población de hombres mayores de 40 años. Los casos de CP de los hospitales de la Sanidad Militar al no tener área asignada sólo computaron para la incidencia de la CM. Para la comparación entre las distintas Áreas Sanitarias ha sido necesario crear un área virtual (Área 12, que atiende a la población de los distritos de Centro-A7 y Arganzuela-A11) cuyo Hospital de referencia es la Fundación Jiménez Díaz. La población de las distintas Áreas Sanitarias después de la creación del Área 12 queda reflejada en la Tabla IV.

$\mathrm{El}$ número total de pacientes con $\mathrm{CP}$ con confirmación patológica durante el año 2000, recogidos en el estudio fue de 2.453. En la Sanidad
TABLA III

RELACIÓN ENTRE LOS HOSPITALES PARTICIPANTES Y EL ÁREA SANITARIA DE LA COMUNIDAD DE MADRID A LA QUE ATIENDEN

\begin{tabular}{||l|l||}
\hline Área sanitaria & Hospitales públicos \\
\hline A1 & H.G.U. Gregorio Marañón \\
\hline A2 & H.U. de La Princesa \\
\hline A3 & H.U. Principe de Asturias \\
\hline A4 & H. Ramón y Cajal \\
\hline A5 & H. La Paz \\
\hline A6 & $\begin{array}{l}\text { Clínica Puerta de Hierro } \\
\text { H. de El Escorial }\end{array}$ \\
\hline A7 & H. Clínico Univ. de San Carlos \\
\hline A8 & $\begin{array}{l}\text { Fundación Hospital Alcorcón } \\
\text { H. de Móstoles }\end{array}$ \\
\hline A9 & H. Severo Ochoa \\
\hline A11 & H. 12 de Octubre \\
\hline $\begin{array}{l}\text { D.S. de Centro (A7) } \\
\text { D.S. de Arganzuela (A11) }\end{array}$ & \begin{tabular}{l} 
Fundación Jiménez Díaz \\
\hline No asignada
\end{tabular} \\
\hline
\end{tabular}

D.S.: distrito sanitario

Pública se diagnosticaron 1.817 (74\%) pacientes: 1.745 en los Hospitales que enviaron sus datos y 72 según la estimación realizada en el Área Sanitaria número 10 (Tabla V). En la Sanidad Privada se diagnosticaron 636 (26\%) pacientes.

La edad media se calculó a partir de los pacientes de la Sanidad Pública que aportaron este dato, 1.732 (95\%) pacientes evaluables. La edad media fue de $70 \pm 7$,8 (40-94) años. El percentil del 25\% fue de 65 , del 50\% (mediana) de 70 y del $75 \%$ de 75 años. La distribución etaria de los casos según decenios: edad desconocida 0,7\%; 40-49 años -0,6\%; 50-59 años -9,6\%; 6069 años $-35,4 \%$; 70-79 años $-43,8 \%$; igual o mayor de 80 años $-9,9 \%$ (Fig. 2).

La incidencia bruta fue de 100,4 casos por 100.00 hombres. La incidencia específica en hombres mayores de 40 años fue de 246,2 y en mayores de 50 años de 367,2 casos por 100.000 hombres. La incidencia específica según decenios fue de: 4,2 casos por 100.000 hombres para el decenio 40-49 años, 86,8 casos para el decenio 50-59 años, 391,8 casos para el decenio 60-69 años, 862,3 casos para el decenio 70-79 años y 


\section{TABLA IV}

POBLACIÓN DE LA COMUNIDAD DE MADRID POR ÁREAS SANITARIAS, CON ESPECIAL ATENCIÓN A LOS HOMBRES DE MÁS DE 40 AÑOS, DESPUÉS DE LA CREACIÓN DEL ÁREA SANITARIA 12 (explicación en el texto)

\begin{tabular}{|c|c|c|c|}
\hline Área sanitaria & Población & Hombres (\%) & Hombres >40 años (\%) \\
\hline A1 & 647.154 & $310.199(47,9 \%)$ & $127.534(41,1 \%)$ \\
\hline A2 & 404.828 & $186.690(46,1 \%)$ & $79.606(42,6 \%)$ \\
\hline A3 & 273.351 & $134.297(49,1 \%)$ & $48.874(36,4 \%)$ \\
\hline A4 & 510.576 & $242.392(47,5 \%)$ & $104.672(43,2 \%)$ \\
\hline A5 & 620.405 & $298.843(48,2 \%)$ & $81.458(40,6 \%)$ \\
\hline A6 & 436.166 & $212.236(48,7 \%)$ & $86.883(45,9 \%)$ \\
\hline A7 & 412.167 & $189.299(45,9 \%)$ & $77.833(38,8 \%)$ \\
\hline A8 & 403.412 & $200.789(49,8 \%)$ & $61.403(35,0 \%)$ \\
\hline A10 & 349.904 & $175.548(50,2 \%)$ & $47.804(37,7 \%)$ \\
\hline A1 & 254.213 & $126.788(49,9 \%)$ & $112.321(42,6 \%)$ \\
\hline A12 & 551.229 & $263.859(47,9 \%)$ & $46.997(45,5 \%)$ \\
\hline Total & 227.931 & $103.327(45,3 \%)$ & $\mathbf{9 9 6 . 2 0 0 ~ ( 4 0 , 8 \% )}$ \\
\hline
\end{tabular}

Los cambios poblacionales en las áreas 7 y 11 se destacan en la tabla en relación con los de la Tabla I.

TABLA V

CASOS DE CÁNCER DE PRÓSTATA DIAGNOSTICADOS EN EL AÑO 2000 EN LA COMUNIDAD DE MADRID SEGÚN LA POBLACIÓN DE LAS DISTINTAS ÁREAS SANITARIAS Y SU DISTRIBUCIÓN POR DÉCADAS DE EDAD

\begin{tabular}{||l|c|c|c|c|c|c|c|c|c|c|}
\hline & \multicolumn{2}{|c|}{ Área 1 } & \multicolumn{2}{c|}{ Área 2 } & \multicolumn{2}{c|}{ Área 3 } & \multicolumn{3}{c|}{ Área 4 } & \multicolumn{2}{c|}{ Área 5 } \\
\hline Edad & Pobl. & C.P. & Pobl. & C.P. & Pobl. & C.P. & Pobl. & C.P. & Pobl. & C.P. \\
\hline $40-49$ & 38.837 & 1 & 26.987 & 0 & 19.984 & 1 & 29.839 & 3 & 41.519 & 1 \\
\hline $50-59$ & 31.970 & 14 & 19.976 & 6 & 14.320 & 10 & 27.371 & 34 & 34.247 & 32 \\
\hline $60-69$ & 32.892 & 95 & 16.702 & 35 & 8.864 & 32 & 28.099 & 99 & 25.549 & 97 \\
\hline $70-79$ & 18.148 & 104 & 11.307 & 45 & 4.226 & 26 & 15.098 & 139 & 14.884 & 154 \\
\hline 80 y más & 5.687 & 13 & 4.724 & 2 & 1.480 & 12 & 4.265 & 29 & 5.259 & 41 \\
\hline Desconocida & & 1 & & & & & & & & 3 \\
\hline Total & $\mathbf{1 2 7 . 5 3 4}$ & $\mathbf{2 2 8}$ & $\mathbf{7 9 . 6 0 6}$ & $\mathbf{8 8}$ & $\mathbf{4 8 . 8 7 4}$ & $\mathbf{8 1}$ & $\mathbf{1 0 4 . 6 7 2}$ & $\mathbf{3 0 4}$ & $\mathbf{1 2 1 . 4 5 8}$ & $\mathbf{3 2 8}$ \\
\hline
\end{tabular}

\begin{tabular}{|c|c|c|c|c|c|c|c|c|c|c|}
\hline \multirow[b]{2}{*}{ Edad } & \multicolumn{2}{|c|}{ Área 6} & \multicolumn{2}{|c|}{ Área 7} & \multicolumn{2}{|c|}{ Área 8} & \multicolumn{2}{|c|}{ Área 9} & \multicolumn{2}{|c|}{ Área 10} \\
\hline & Pobl. & C.P. & Pobl. & C.P. & Pobl. & C.P. & Pobl. & C.P. & Pobl. & C.P.(*) \\
\hline $40-49$ & 30.644 & 0 & 22.008 & 1 & 30.872 & 0 & 28.593 & 2 & 18.476 & 1 \\
\hline $50-59$ & 22.871 & 11 & 23.412 & 7 & 25.375 & 19 & 18.160 & 15 & 15.932 & 8 \\
\hline 60-69 & 15.617 & 30 & 22.184 & 41 & 12.784 & 60 & 9.382 & 36 & 8.214 & 32 \\
\hline $70-79$ & 8.317 & 33 & 13.594 & 74 & 6.284 & 52 & 3.938 & 18 & 3.817 & 24 \\
\hline 80 y más & 3.366 & 10 & 5.685 & 17 & 2.518 & 13 & 1.330 & 4 & 1.311 & 7 \\
\hline Desconocida & & & & 2 & & & & & & \\
\hline Total & 80.815 & 84 & 86.883 & 142 & 77.833 & 144 & 61.403 & 75 & 47.804 & 72 \\
\hline
\end{tabular}

(*)Casos estimados en función de las Áreas 3, 6 y 8 (explicación en el texto).

\begin{tabular}{|l|c|c|c|c|c|}
\hline & \multicolumn{2}{|c|}{ Área 11 } & \multicolumn{2}{c|}{ Área 12 } \\
\hline Edad & Pobl. & C.P. & Pobl. & C.P. \\
\hline $40-49$ & 30.256 & 0 & 14.814 & 0 \\
\hline $50-59$ & 27.503 & 10 & 10.685 & 1 \\
\hline $60-69$ & 31.468 & 44 & 10.563 & 31 \\
\hline $70-79$ & 17.782 & 75 & 7.560 & 31 \\
\hline 80 y más & 5.312 & 23 & 3.375 & 4 & 3 \\
\hline Desconocida & & 3 & & \\
\hline Total & $\mathbf{1 1 2 . 3 2 1}$ & $\mathbf{1 5 5}$ & $\mathbf{4 6 . 9 9 7}$ & $\mathbf{7 0}$ \\
\hline
\end{tabular}

(\$)Los Hospitales Militares no tienen asignada un Área Sanitaria específica (explicación en el texto). 


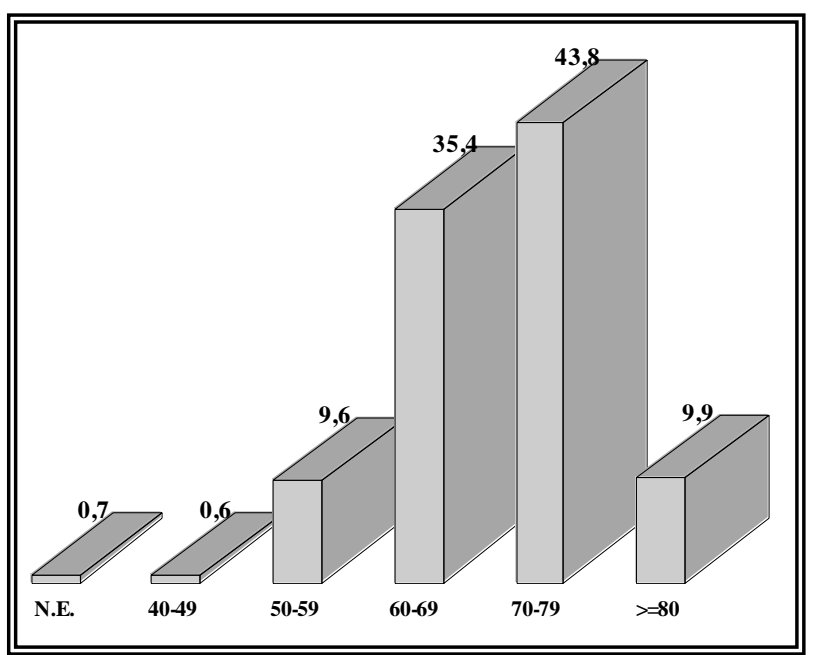

FIGURA 2. Distribución por decenios en porcentajes de los pacientes con cáncer de próstata diagnosticados en la Comunidad de Madrid en el año 2000. NE: edad no especificada.

de 550,6 casos en los hombres con 80 años o más (Fig. 3). La incidencia ajustada a la población española fue de 120,1 casos por 100.000 hombres. La incidencia ajustada a la población estandarizada europea y mundial fue de 103,5 casos y de 68,6 casos por 100.000 hombres respectivamente.

La distribución por decenios de los pacientes diagnosticados de CP según las distintas Áreas Sanitarias se refleja en la Figura 4. Observamos que en la mayoría de las Áreas el porcentaje más elevado de casos se sitúa en el decenio de 70-79 años, excepto en las Áreas 3, 8, 9 y 10 que se localizan en el decenio de 60-69 años. La edad de

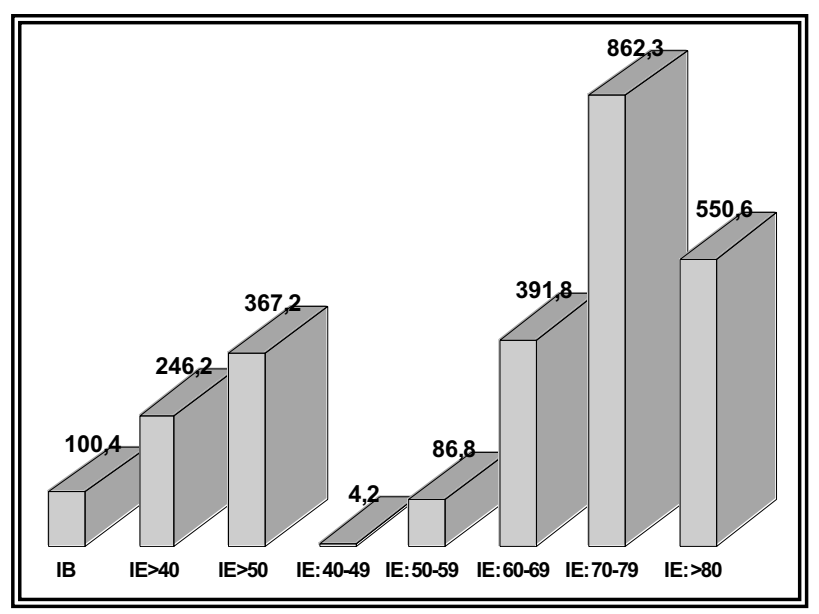

FIGURA 3. Incidencia bruta (IB) $e$ incidencia especifica (IE) (casos $x 100.000$ varones) por edades del cáncer de próstata en la Comunidad de Madrid en el año 2000.

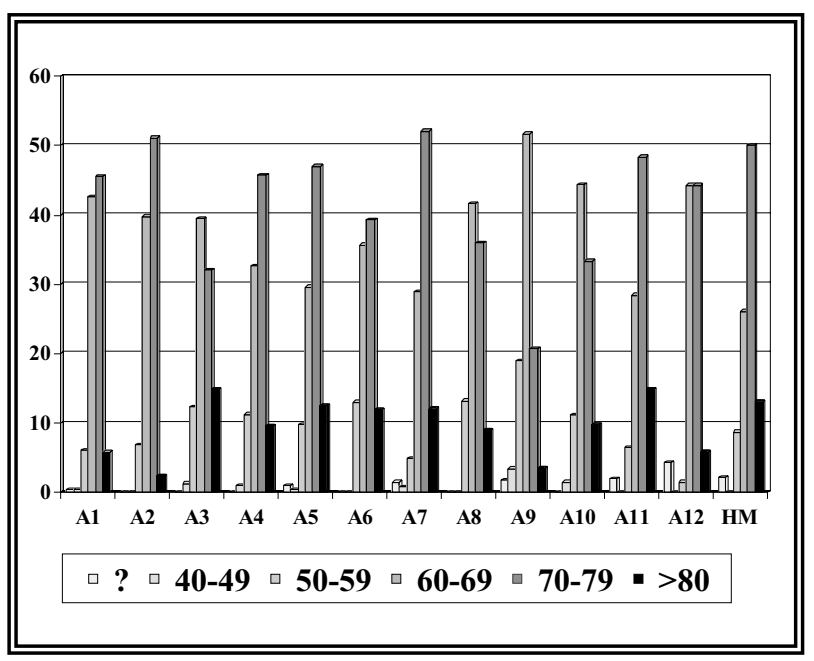

FIGURA 4. Comparación (en porcentaje) según decenios y Áreas Sanitarias de los pacientes con cáncer de próstata diagnosticados en la Comunidad de Madrid en el año 2000. ? - edad desconocida.

los pacientes con CP según las distintas Áreas Sanitarias se resumen en la Tabla VI (Fig. 5). La mediana de edad más baja se obtuvo en el área 9 (65 años) y la más elevada en el área 11 (72 años) y en los Hospitales militares (73 años).

La incidencia bruta y específica por edades para cada Área se detalla en la Tabla VII. La incidencia ajustada a la población de la CM según las distintas Áreas Sanitarias se detalla en la Tabla VIII. Las áreas 4 (113 por 100.000 hombres) y 5 (112 por 100.000 hombres) tiene la incidencia ajustada más elevada. La incidencia más baja se observó en las áreas 2 y 6, ambas con 46 por 100.000 hombres En la Figura 6 se comparan la incidencia bruta y la incidencia ajustada según la edad de las Áreas Sanitarias. En las Áreas Sanitarias en las que existía un consenso para pedir de forma sistemática PSA a todo varón mayor de 50 años que acudía a la consulta de Urología, la incidencia ajustada oscilaba entre 78,2 y 113,4 casos por 100.000 varones, mientras que en las Áreas donde el PSA se determinaba en función de las preferencias personales de cada Urólogo la incidencia oscilaba entre 45,7 y 65,4 casos por 100.000 (Fig. 7).

\section{DISCUSIÓN}

El cáncer de próstata es probablemente la enfermedad oncológica más prevalente. En series de autopsias entre el $30 \%$ y el $40 \%$ de los hombres 


\section{TABLA VI}

EDAD DE LOS PACIENTES DIAGNOSTICADOS DE CÁNCER DE PRÓSTATA EN LAS DISTINTAS ÁREAS SANITARIAS

DE LA COMUNIDAD DE MADRID EN EL AÑO 2000 (no se incluye el área 10 ya que sus casos fueron estimados)

\begin{tabular}{|c|c|c|c|c|c|c|c|c||}
\hline Área & N & PE & E.M. & D.S. & Min-Máx. & P-25\% & P-50\% & P-75\% \\
\hline A1 & 228 & 227 & 69,4 & 7,03 & $49-89$ & 65 & 70 & 75 \\
\hline A2 & 88 & 88 & 69,4 & 6,22 & $52-81$ & 65 & 70 & 75 \\
\hline A3 & 81 & 81 & 69,2 & 9,12 & $49-87$ & 61,5 & 68 & 77,5 \\
\hline A4 & 304 & 304 & 69,6 & 8,03 & $40-91$ & 64 & 70 & 76 \\
\hline A5 & 328 & 325 & 70,9 & 7,85 & $40-91$ & 66 & 71 & 76 \\
\hline A6 & 84 & 84 & 69,3 & 8,73 & $50-88$ & 65 & 70 & 76 \\
\hline A7 & 142 & 140 & 71,5 & 7,36 & $49-89$ & 68 & 71 & 77 \\
\hline A8 & 144 & 144 & 69,1 & 7,55 & $53-85$ & 64 & 69 & 75 \\
\hline A9 & 75 & 75 & 65,8 & 7,80 & $47-85$ & 60 & 65 & 70 \\
\hline A11 & 155 & 152 & 72,1 & 7,94 & $51-93$ & 67 & 72 & 78 \\
\hline A12 & 70 & 67 & 69,6 & 5,21 & $57-82$ & 66 & 70 & 72 \\
\hline H.M. & 46 & 45 & 70,8 & 8,34 & $51-94$ & 64 & 73 & 76 \\
\hline
\end{tabular}

N: número de casos. PE: pacientes evaluables. E.M.: edad media. D.S.: desviación estándar. Min-Máx.: valores mínimo y máximo. P: percentiles al 25\%, 50\% (mediana) y 75\%. H.M.: hospitales militares.

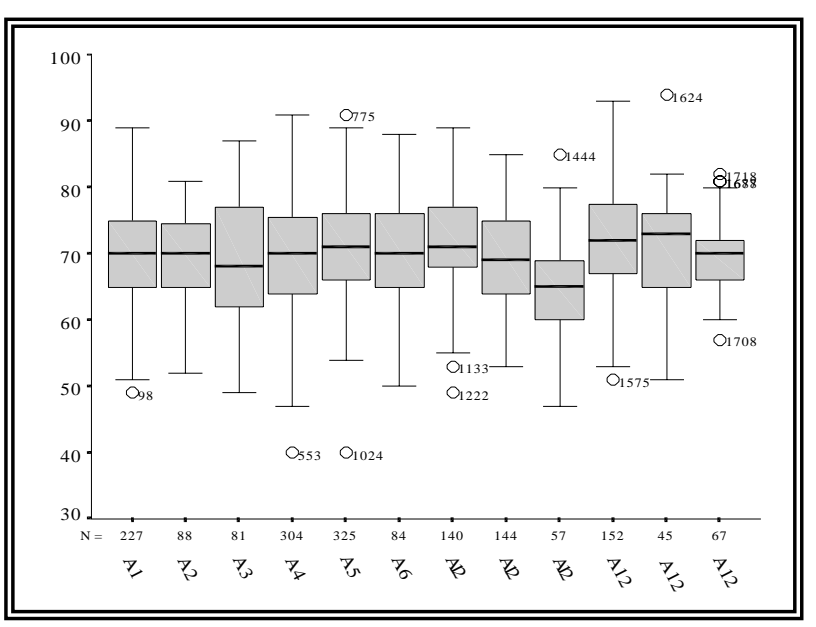

FIGURA 5. Comparación de la edad entre las distintas Áreas Sanitarias de los pacientes con cáncer de próstata diagnosticados en la Comunidad de Madrid en el año 2000. La linea gruesa del interior de la caja corresponde a la mediana. El limite superior de la caja se corresponde con el percentil $75 \%$ y el inferior con el $25 \%$, por lo tanto la caja engloba al $50 \%$ de los casos centrales. La linea horizontal superior corresponde a la edad más elevada y la inferior a la más baja que no llegan a ser atipicas. Los circulos corresponden a los casos atipicos.

mayores de 50 años presentan evidencia histológica de la enfermedad ${ }^{8,9}$ y aproximadamente el $70 \%$ de los hombres mayores de 70 años ${ }^{10}$, aunque sólo un 25\% de los sujetos desarrollará la enfermedad clínica y únicamente el 7-8\% morirá por su causa ${ }^{8,9}$.
El número de sujetos con diagnóstico histológico de cáncer de próstata, y por lo tanto su incidencia en una población, vendrá determinada por los siguientes factores: edad, raza, utilización libre o restringida por parte de los médicos del PSA como marcador tumoral, criterios (niveles de PSA, edad límite, etc.) para la indicación de la biopsia de la glándula y la agresividad de la técnica de biopsia (cuanto mayor sea el número de cilindros obtenidos por paciente más alta es la probabilidad de detectar un cáncer de próstata oculto o subclínico). La edad y la raza son factores de riesgo no modificables, pero el resto de factores comentados podrían contribuir a explicar las diferencias encontradas en la incidencia del $\mathrm{CP}$ en poblaciones similares.

La incidencia bruta del CP en la Comunidad de Madrid fue de 100,4 casos por 100.000 hombres. En 1997 Arizabalaga y cols. ${ }^{5}$ publicaron la evolución de la incidencia del $\mathrm{CP}$ en el Área Sanitaria $\mathrm{n}^{\circ} 8$ de la Comunidad de Madrid en el periodo 1983-1996, comunicando un aumento progresivo de la incidencia hasta 36,5 casos por 100,000 hombres en 1996. Si comparamos estos datos con los de nuestro estudio observamos que la incidencia en este mismo área se ha duplicado (71,7 casos $x 100.000$ hombres) en un periodo de 4 años. 
TABLA VII

INCIDENCIA BRUTA (CASOS X 100.000 VARONES) E INCIDENCIA ESPECÍFICA POR EDADES DEL CÁNCER DE PRÓSTATA EN EL AÑO 22000 EN LAS DISTINTAS ÁREAS SANITARIAS DE LA COMUNIDAD DE MADRID

\begin{tabular}{|c|c|c|c|c|c|c|c|c|c||}
\hline Área & $\mathbf{N}$ & IB & IE $>$ 40 & IE $>50$ & IE40-49 & IE50-59 & IE60-69 & IE70-79 & IE $\geq \mathbf{8 0}$ \\
\hline A1 & 228 & 73,5 & 178,8 & 255,9 & 2,6 & 43,8 & 288,8 & 573,1 & 228,6 \\
\hline A2 & 88 & 47,1 & 110,5 & 167,2 & 0 & 30,0 & 209,6 & 398,0 & 42,3 \\
\hline A3 & 81 & 60,3 & 165,7 & 276,9 & 5,0 & 69,8 & 361,0 & 615,2 & 810,8 \\
\hline A4 & 304 & 125,4 & 290,4 & 402,2 & 10,0 & 124,2 & 352,3 & 920,6 & 679,9 \\
\hline A5 & 325 & 108,8 & 267,6 & 409,1 & 2,4 & 93,4 & 379,7 & 1034,7 & 779,6 \\
\hline A6 & 84 & 39,6 & 103,9 & 167,4 & 0 & 48,1 & 192,1 & 396,8 & 297,1 \\
\hline A7 & 142 & 75,0 & 163,4 & 217,3 & 4,5 & 29,9 & 184,8 & 544,3 & 299,0 \\
\hline A8 & 144 & 71,7 & 185,0 & 306,6 & 0 & 74,9 & 469,3 & 827,5 & 516,3 \\
\hline A9 & 75 & 42,7 & 122,1 & 222,5 & 7,0 & 882,6 & 383,7 & 457,1 & 300,7 \\
\hline A10 $*$ ) & 72 & 56,8 & 150,6 & 242,1 & 5,4 & 50,2 & 389,6 & 628,8 & 533,9 \\
\hline A11 & 155 & 58,7 & 138,0 & 188,9 & 0 & 36,4 & 139,8 & 421,8 & 433,0 \\
\hline A12 & 70 & 67,7 & 148,9 & 217,5 & 0 & 9,3 & 293,5 & 410,0 & 118,5 \\
\hline
\end{tabular}

(*)Casos estimados en función de las áreas 3, 6 y 8. N: casos de CP por área. IB: incidencia bruta. IE: incidencia específica según la edad.

\section{TABLA VIII}

INCIDENCIA AJUSTADA E INCIDENCIA ESPECÍFICA AJUSTADA POR LA EDAD (CASOS X 100.000 VARONES) SEGÚN LA POBLACIÓN DE LA COMUNIDAD DE MADRID EN LOS PACIENTES DIAGNOSTICADOS DE CÁNCER DE PRÓSTATA EN LAS DISTINTAS ÁREAS SANITARIAS DE LA COMUNIDAD DE MADRID EN EL AÑO 2000

\begin{tabular}{||c|c|c|c|c|c|c|c|c|c||}
\hline Área & $\mathbf{N}$ & IAE & IAE $\geq \mathbf{4 0}$ & IAE $\geq \mathbf{5 0}$ & IAE40-49 & IAE50-59 & IAE60-69 & IAE70-79 & IAE $\geq \mathbf{8 0}$ \\
\hline A1 & 203 & 65,4 & 159,2 & 237,8 & 2,3 & 43,1 & 288,1 & 574,9 & 229,1 \\
\hline A2 & 86 & 46,1 & 108,0 & 162,2 & 0 & 32,2 & 208,3 & 400,4 & 56,5 \\
\hline A3 & 105 & 78,2 & 214,8 & 319,5 & 6,1 & 67,5 & 357,6 & 619,6 & 828,0 \\
\hline A4 & 275 & 113,4 & 262,7 & 388,7 & 11,4 & 126,0 & 351,0 & 921,2 & 687,3 \\
\hline A5 & 335 & 112,1 & 275,8 & 411,7 & 4,9 & 93,5 & 380,0 & 1030,1 & 777,5 \\
\hline A6 & 97 & 45,7 & 120,0 & 180,2 & 0 & 49,9 & 194,1 & 394,4 & 306,0 \\
\hline A7 & 115 & 60,8 & 132,4 & 197,0 & 3,4 & 29,5 & 185,7 & 541,2 & 310,5 \\
\hline A8 & 197 & 98,1 & 253,1 & 380,0 & 0 & 75,3 & 472,1 & 829,3 & 519,9 \\
\hline A9 & 101 & 57,5 & 164,5 & 304,8 & 4,9 & 83,6 & 386,8 & 454,3 & 292,9 \\
\hline A10(*) & 98 & 77,3 & 205,0 & 304,7 & 6,3 & 53,7 & 393,7 & 616,8 & 517,4 \\
\hline A1 1 & 127 & 48,1 & 113,1 & 169,8 & 0 & 35,9 & 139,6 & 418,6 & 440,4 \\
\hline A12 & 59 & 57,1 & 125,5 & 188,5 & 0 & 7,8 & 343,2 & 356,1 & 95,7 \\
\hline
\end{tabular}

$\mathrm{N}$ : casos esperados. IAE: incidencia ajustada según la edad. HM: hospitales militares. (*)Casos estimados en función de las áreas 3, 6 y 8.

Existen muy pocos datos sobre la incidencia del CP en otras regiones o Comunidades Autónomas con la actualidad suficiente para que puedan ser comparadas con nuestro estudio. Recientemente Caballero y cols. ${ }^{11}$ publicaron la evolución de la incidencia del CP en el periodo 1996-1999 en la zona de influencia del Hospital Costa del Sol de Marbella (Málaga), encontraron un aumento de la incidencia de 18,7 a 28,7 casos por 100.000 en el periodo de estudio. En la
Comunidad Valenciana la incidencia de CP ajustada a la población mundial para los años 19901994 fue de 35,79 por 100.000 hombres. La distribución por provincias fue muy distinta: Alicante con 34,81 , Castellón con 68,78 y Valencia con 21,14 (fuente: http://dgsp.san.gva.es/ sscc/epidemiologia/tumores/tumores.htm). En nuestro estudio la incidencia ajustada a la población mundial fue de 68,6 casos por 100.00 varones. 


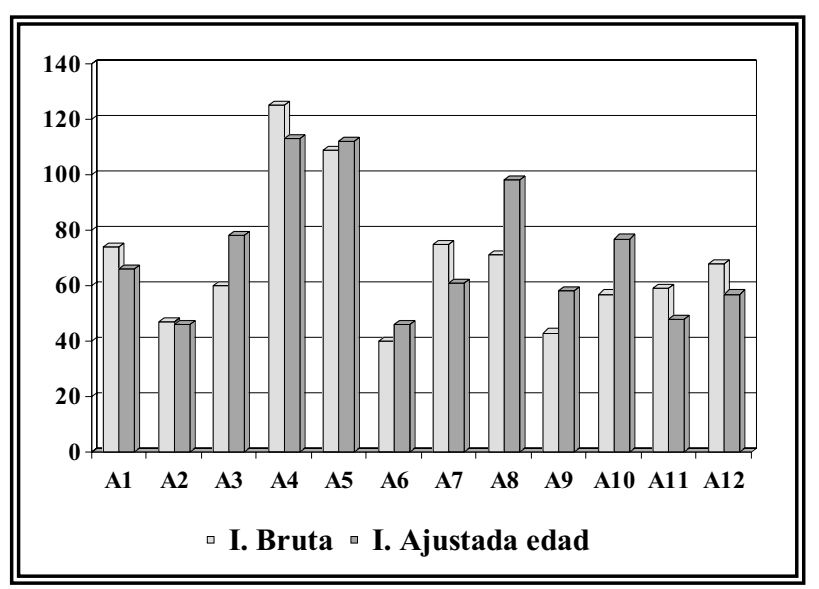

FIGURA 6. Comparación entre la incidencia bruta y la incidencia ajustada por la edad según de las distintas Áreas Sanitarias en los pacientes con cáncer de próstata diagnosticados en la Comunidad de Madrid en el año 2000.

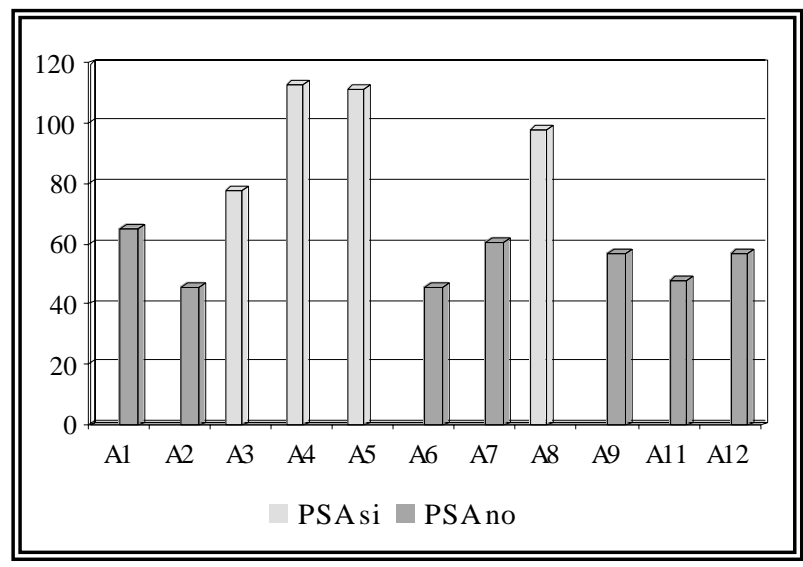

FIGURA 7. Comparación entre la incidencia ajustada por la edad y la existencia de consenso para pedir sistemáticamente PSA a todos los varones mayores de 50 años (PSA si) o pedirlo según criterios personales (PSA no) en las distintas Áreas Sanitarias en los pacientes con cáncer de próstata diagnosticados en la Comunidad de Madrid en el año 2000.

La incidencia del CP en los registros de tumores de otros países europeos es muy variable. En la región de Rotterdam en la que se desarrolla un programa de screening (European Randomised Screening study for Prostate Cancer - ERSPC) ${ }^{12}$, Spapen y cols. ${ }^{13}$ comunicaron una incidencia del CP ajustada a la población europea en el año 1995 de 125 casos por 100.000 varones de los cuales sólo el 16\% de los casos fueron diagnosticados dentro del programa de screening. A través de Internet es posible acceder a varios registros de tumores europeos (http://www.ikr.nl/canregs.htm). La incidencia ajustada a la población europea fue: en Bélgica para 1997 de 87,8 casos por 100.000 hombres; en Finlandia para 1998 de 77,5 casos por 100.000 hombres; en Irlanda para 1998 de 77,5 casos por 100.000 hombre y en Holanda de 84,5 casos por 100.000 hombres.

La diferencia observada entre las incidencias obtenidas en los registros de tumores y la de nuestro estudio es difícil de explicar, pero las tres razones siguientes podrían contribuir a ello:

1. Desconocemos la metodología utilizada en los registros de tumores, pero es muy probable que la incidencia no sea la real sino la estimada en función de una recogida parcial de datos.

2. En nuestro estudio se incluyen los casos diagnosticados en la Sanidad Privada que suponen un $26 \%$ de los mismos. Ignoramos si también se incluyen en los registros de tumores, pero suponemos que no.

3. Los diferentes criterios clínicos utilizados en el diagnóstico de los pacientes.

La incidencia del CP en USA según el programa SEER (Surveillance, Epidemiology, and End Results) del NCI (National Cancer Institute) (http://www.seer.cancer.gov/Publications/ProstM ono/citation/html) para el periodo 1994-1998 fue de 142 casos por 100.000 varones (ajustada a la población estándar de USA de 1970) y la incidencia para el mismo periodo ajustada a la población mundial fue de 109 casos por 100.000 hombres.

En USA la incidencia del CP ha evolucionado con el tiempo. En el periodo 1973-1988 la incidencia del CP experimentó un incremento anual del 2,9\%. Después de la introducción del PSA la incidencia aumentó de forma muy importante, un $17,5 \%$ por año entre 1988 y 1992 . A partir de aquí la incidencia comenzó a decaer aunque no de una forma global, en el periodo 1992-1995 descendió un 10\% anual estabilizándose entre 1995 y 1998 (sólo cambios del 0,3\%) ${ }^{14}$. Los pocos datos existentes parecen apuntar que en Europa nos encontramos actualmente en un periodo de incremento de la incidencia del CP. Murphy y cols. ${ }^{10}$ comunicaron un incremento del $30 \%$ en la incidencia del CP en la región de Yorkshire en el periodo 1981-1990. Según Brewster y cols. ${ }^{15}$ la incidencia en Escocia aumentó un 59\% en el periodo 1982-1996. Según el registro de tumores de Rotterdam ${ }^{13}$, la incidencia del CP entre 1989 y 
1995 aumentó de 62 a 125 casos por 100.000 hombres. La incidencia en Inglaterra y Gales en el periodo 1971-1993 experimentó un aumento del 104\%, pasando de 29 a 59 casos por 100.000 hombres. En el Área Sanitaria número 8 de la Comunidad de Madrid la incidencia del CP durante el periodo 1983-1996 aumentó desde un 3,7 a 36,5 casos por 100.000 hombres $^{5}$. Como ya hemos comentado en nuestro estudio la incidencia de este mismo Área fue de 71,7 casos por 100.000 hombres, lo que supone un incremento del $97 \%$ en cuatro años.

En las estimaciones publicadas para la Unión Europea en $1995^{2}$ sobre la incidencia estimada de distintas enfermedades oncológicas, a las que nos referimos con anterioridad, el cáncer de próstata ocupaba el segundo puesto en frecuencia después del cáncer de pulmón. En el trabajo de Spapen sobre el registro de Rotterdam ${ }^{13}$ se comunica que la incidencia del CP es superior a la del cáncer de pulmón. En algunos de los registros de tumores con acceso a través de Internet como Bélgica (Limburg) o Francia la incidencia del CP es superior a la del cáncer de pulmón. En España no existen datos sobre la incidencia del cáncer de pulmón. La incidencia bruta del cáncer broncopulmonar en la Comunidad de Castilla y León en el año 1997 fue de 73,04 casos por 100.000 hombres y de 66,79 cuando se ajustó a la población española de $1991^{16}$. Por lo tanto, es muy probable que el CP sea ya el cáncer más frecuente del hombre en España.

Al comparar la incidencia ajustada según la edad (Tabla VIII) se observa una incidencia más elevada en las Áreas $4(113,4$ por 100.000), 5 $(112,1$ por 100.000$)$ y $8(98,1$ por 100.000$)$. Las Áreas 4 y 5 presentan sobre todo una incidencia más elevada en el periodo entre 70-79 años con 921,2 y $1.030,1$ por 100.000 hombres respectivamente. El Área 8 presenta una elevada incidencia en los periodos entre 60-69 años (472,1 por 100.000) y $70-79$ años (829,3 por 100.000). En cambio en las Áreas $2(46,1), 6(45,7)$ y $11(48,1)$ la incidencia está por debajo de 50 casos por 100.000 hombres. Estas diferencias no son explicables por diferencias raciales y sí pueden serlo por diferencias en la sistemática diagnóstica. Los datos extraídos de la encuesta a los Investigadores confirman que las Áreas que realizan PSA de forma sistemática a todos los varones mayores de 50 años que acudían a la consulta, presentaron una incidencia ajustada muy superior (entre 78,2 y 113,4 casos por 100.000 hombres) que la de las Áreas que no seguían la misma sistemática (entre 45,7 y 65,4 casos por 100.000 hombres).

El 26\% de los sujetos han sido diagnosticados en la Sanidad Privada. Esto podría contribuir también a explicar las diferencias en la incidencia entre las distintas Áreas sanitarias. Para ello sería necesario que la incidencia de diagnóstico en la Sanidad Pública en las Áreas sanitarias con un nivel económico más alto fuera más baja que en el resto. El nivel socio-económico influye directamente en el estado de salud de una comunidad. Existen trabajos en la literatura en los que la asociación entre el nivel socio-económico y el cáncer de próstata es variable (positiva, negativa o no asociación) ${ }^{17,18}$. Recientemente Liu y cols. ${ }^{18}$ encuentran que existe una asociación entre el nivel socio-económico y el $\mathrm{CP}$, debido a que la población con un alto nivel socio-económico es más prevalente en los screening poblacionales ya que esta subpoblación tiene un mejor acceso a los cuidados médicos. En nuestra Comunidad toda la población tiene en teoría el mismo nivel de accesibilidad a los Servicios Sanitarios, pero los sujetos con un nivel socio-económico más alto además de preocuparse más por su estado de salud, suelen tener con mayor frecuencia contratados seguros sanitarios privados y, por lo tanto, un acceso más rápido a los servicios sanitarios. La influencia que los diagnósticos en la Sanidad Privada puedan ejercer sobre las diferencias en la incidencia en las distintas Áreas Sanitarias es muy difícil de demostrar, ya que desconocemos el domicilio de los pacientes diagnosticados en la Sanidad Privada. Por otra parte, no existen indicadores económicos por áreas sanitarias. Existe en: http://www.comadrid.es/iestadis/irfdm97.htm información sobre la renta familiar disponible municipal, pero la ciudad de Madrid la trata como un municipio único, por lo tanto no es posible averiguar con estos datos la renta según las áreas sanitarias.

Aunque estamos convencidos de que la incidencia del CP obtenida en este estudio tiene una fiabilidad muy alta, existen una serie de circunstancias que pudieran producir ligeras variaciones (aumentarla o disminuirla) en la misma. Las circunstancias que puede elevar la incidencia son: 
1. La estimación de los casos del Área 10 puede que sea inferior a la real, ya que el Hospital de Getafe participa en el ERSPC.

2. No hemos recogido los casos de CP incidentales diagnosticados en la Sanidad Privada.

3. Es probable que no hayamos recogido todos los casos diagnosticados mediante biopsia ecodirigida en la Sanidad Privada.

4. El Hospital de la Cruz Roja realiza intervenciones quirúrgicas por hiperplasia benigna de próstata y sus casos de $\mathrm{CP}$ incidentales no se han recogido al no participar este Centro en el estudio. Por otra parte, un porcentaje de pacientes censados en otras Comunidades Autónomas (generalmente limítrofes) son diagnosticados en nuestra Comunidad aprovechando la residencia de algún familiar (generalmente hijos) en ella.

En conclusión, la incidencia bruta del CP en la Comunidad de Madrid en el año 2000 fue de 100,4 casos por 100.000 hombres. La incidencia ajustada a la población española, europea y mundial fue de 120,1, 103,5 y 68,6 casos por 100.000 hombres respectivamente. Existe una diferencia en la incidencia entre las distintas Áreas Sanitarias que parece justificarse por la variabilidad en el uso del PSA, aunque no podemos decir que el elevado porcentaje de diagnósticos en la Sanidad Privada no haya podido influir también. El 26\% de los pacientes se diagnosticó en la Sanidad Privada, hecho a tener muy en cuenta a la hora de diseñar futuros estudios poblacionales en esta patología.

Agradecimientos: a los Drs. Teodoro Mayayo Dehesa, Javier de la Riva de la Viña, Pedro Paños Lozano, Carlos Roque Mir y Eduardo Martín Oses, Enrique Pérez-Castro Ellendt y Jorge Massarrah Halabi y Alfredo Hernández Villaverde que han aportado su casuística privada. A los Dres. Gregorio Garrido Cantarero, Dolores Vigil Escribano y Paz Rodríguez Pérez de la Unidad de Investigación Clínico-Epidemiológica del H.G.U. Gregorio Marañón.

\section{REFERENCIAS}

1. GREENLEE RT, HILL-HARMON MB, MURRAY T, THUN M.: Cancer statistics, 2001. CA Cancer J Clin 2001; 51: 15-36.

2. BRAY F, FERLAY RS, PARKIN DM.: Estimates of cancer incidence and mortality in Europe in 1995. Eur $J$ Cancer 2002; 38: 99-166.
3. RUIZ LISO JM, SANZ ANQUELA JM, ALFARO TORRES J, GARCÍA PÉREZ MA, DODERO DE SOLANO E.: Tendencias de mortalidad e incidencia neoplásica urológica (próstata, vejiga y riñón). Soria 19501988. Arch Esp Urol 1989; 42: 532-538.

4. OTERO MAURICIO G, FERNÁNDEZ FERNÁNDEZ A, GIL FABRA J.: Epidemiología descriptiva y analítica en oncología urológica. Tema Monográfico. LXIII Congreso Nacional de Urología. 1998. ENE ediciones. Madrid.

5. ARRIZABALAGA MORENO M, GARCÍA GONZÁLEZ JI, DÍEZ RODRÍGUEZ JM y cols.: Indicadores epidemiológicos del adenocarcinoma de próstata. Resultados sobre 436 pacientes. Actas Urol Esp 1997; 21: 852861.

6. Mapa Sanitario Comunidad de Madrid. Comunidad de Madrid. Consejería de Sanidad y Servicios Sociales. ISBN 84-451-1545-6. 1999.

7. COLTON T.: Estadística en Medicina. Masson-Salvat Medicina. Barcelona, 1991.

8. SCARDINO PT, WEAVER R, HUDSON MA.: Early detection of prostate cancer. Hum Patol 1992; 23: 211-222.

9. SEIDMAN H, MUSHINSKI MH, GELB SK, SILVERBERG E.: Probabilities of eventually developing or diying of cancer. United States, 1985. CA Cancer $J$ Clin 1985; 35: 35-56.

10. MURPHY M, JOHSTON C, WHELAN P, RIDER L, LLOYD SN.: Changing trends in prostatic cancer. BJU International 1999; 83: 786-791.

11. CABALLERO ALCÁNTARA J, MARCHAL ESCALÓN C, PADILLA LEÓN M, RODRIGO FERNÁNDEZ I.: Aumento de la tasa cruda de la incidencia del cáncer de próstata en nuestro medio. Actas Urol Esp 2001; 25: 624-627.

12. SCHRÖDER FH, BANGMAN CH.: The European randomised study of screening for prostate cancer (ERSPC). Br J Urol 1997; 79: 68-71.

13. SPAPEN SJJC, DAMHUIS RAM, KIRKELS WJ.: Trends in the curative treatment of localized prostate cancer after the introduction of prostate-specific antigen: data from the Rotterdam cancer registry. BJU International 2000; 85: 474-480.

14. SARMA AV, SCHOTTENFELD D.: Prostate cancer incidence, mortality, and survival trends in the Unitate States: 1981-2001. Sem Urol Oncol 2002; 20: 3-9.

15. BREWSTER DH, FRASER LA, HARRIS V, BLACK RJ.: Rising incidence of prostate cancer in Scotland: increased risk or increased detection?. BJU International 2000; 85: 463-473.

16. Sociedad Castellano-Leonesa de Patología Respiratoria (SOCALPAR). El carcinoma broncopulmonar en la Comunidad de Castilla y León, año 1997. FAES.

17. NILSEN TL, JOHNSEN R, VATTEN LJ.: Socio-economic and lifestyle factors associated with the risk of prostate cancer. Br J Cancer 2000; 82: 1358-1363.

18. LIU L, COZEN W, BERNSTEIN L, ROSS RK, DEAPEN D.: Changing relationship betwen socioeconomic status and prostate cancer incidence. $J$ Nat Cancer Inst 2001; 93: 705-709.

Dr. F. Herranz Amo

C/ Doctor Esquerdo, 155 $5^{\underline{a}}-7^{\circ} 3$

28007 Madrid

(Trabajo recibido el 19 noviembre de 2002) 